\title{
Brazilian Society of Infectious Diseases: 25 Years!
}

Infectious Diseases (ID) is possibly the branch of Medicine that has contributed the most in the twentieth century towards mankind's struggle for survival against life-threatening diseases caused by a specific microorganism. All diseases have a specific etiology but ID involve definite targets that have to be or should be attained in order to develop strategies for the elimination of the causative agent or, ideally, vaccines for the prevention of the disease. Centuries and millennia have been individually marked by devastating epidemics caused by a specific microorganism. Cholera and plague in the past, and the influenza epidemics, malaria, tuberculosis, viral hepatitis and HIV infection, among others of the present day, are some of the diseases that have been responsible for the deaths of millions of the "kings of the mammalian species."

The concept of the Hippocratic Art of Medicine changed dramatically in the twentieth century thanks to the highly sophisticated scientific activity that was developed over that period to protect the health of the various species on the planet. Human Medicine has changed dramatically since Hippocrates' time when it was possible for one single physician to be competent in all fields of medicine. Until relatively recently, it was common to see a certificate on a physician's office wall approving him to practice Medicine as a "Doctor of Surgery and Clinical Sciences". Today, this kind of certificate would not be a good reference for a doctor. A modern day physician needs to prove to his patients and colleagues that he is authorized to practice a certain medical specialty and that he has achieved a certain scientific degree.

The Brazilian Society of Infectious Diseases was created on January 30, 1980. The primary objective of the Society was to provide opportunities in Brazil for those practicing or wishing to practice the specialty of ID. Initially, the Society was registered as the Brazilian Society of Infectious Diseases. However, the name was changed to the Brazilian Society of Infectology (SBI) in the bylaws of February 5, 1980, and the first President of the Society was Professor
Ricardo Veronesi. Thirty-seven members signed this document. However, one historical aspect regarding societies of infectious disease in Brazil should be emphasized. Tropical diseases are endemic in this country. Physicians and other specialists working in public healthcare and specifically in infectious diseases got together to form the Brazilian Society of Tropical Medicine, which was created ten years prior to the SBI. Although the concept of infectious diseases in Brazil is the same as that in Europe, these parasitic diseases predominantly affect humans in the tropical regions of the world. Continental colonization by Europeans in the past and in some areas even today led to the foundation of well-known, reputable universities and academic centers of excellence that formally consolidated the study of infectious diseases in the areas most affected by the problems caused by tropical diseases.

The two decades stretching from 1970 to 1990 definitively changed this concept. The current search for antibiotics to overcome bacterial resistance to penicillin and to a few other antimicrobial agents available, led to an explosion of new, potent antibiotic agents for the treatment of bacterial infection. It has become extremely complex for family practitioners, surgeons and specialists in other fields of Medicine to prescribe an antimicrobial agent without the advice of a specialist in ID. Nosocomial infections, vaccines and emergent infectious diseases have become an activity exclusively reserved for well-trained healthcare workers with post-graduate training in ID. Several renowned scientific journals, such as Nature and Lancet, have sister-journals dedicated to publications on ID, while there are also some new, highly specialized journals that focus on the different fields within the specialty of Infectious Diseases, such as mycology, microbiology, virology and, more recently, HIV infection and AIDS. Since its creation many years ago, The Infectious Diseases Society of America has been the major source of Continuing Medical Education (CME) on ID for most physicians graduating in the US. 
In Brazil, healthcare workers and physicians specializing in ID were allocated to the various institutes of Tropical Diseases and academic organizations in the country, such as the Schools of Medicine of the federal and state universities, or specific government organizations dealing with public health and specifically with the principal tropical diseases prevalent in the country. The Brazilian Society of Infectious Diseases became the most important organization of ID specialists in Brazil to respond to the HIV epidemic. Most of the activities of the Society are related to providing education and opportunity for ID specialists around the country to update their knowledge on the management of opportunistic infections and antiretroviral therapy. In addition, the reemergence of old, endemic IDs in this country, such as dengue, yellow fever, tuberculosis, malaria and cholera, has alerted the Brazilian Society of Medicine to the need to recognize ID as a formal specialty that should be included in the medical curricula, requiring, in addition, specific postgraduate medical training at Residency level, leading to a specialist title in ID. This decision caused a great impact on the Brazilian Society of Infectious Diseases, which was formerly referred to as the Brazilian Society of Infectology. In its relatively short period of existence of 25 years, this society has grown from an original 37 member physicians to more than 1,000 ID specialists in 23 states of Brazil. In addition, the SBI has placed strong emphasis on education and scientific activity by creating its own scientific journal, The Brazilian Journal of Infectious Diseases (BJID) in 1997, thanks to the praiseworthy dedication of its deputy editors-in-chief, Roberto Badaró and Thomas C. Jones. This journal now takes its place among the scientific journals originating from Brazil and from around the world that have a high impact on scientific medical information. It is indexed on Medline and in the Index of Current Scientific Information. A new editor has already been selected to replace Professor Badaró in his duties of coordinated peer review of the papers submitted to the BJID. Dr. Anastacio Queiroz de Souza is a specialist in ID with an irrefutable scientific reputation both in Brazil and worldwide for his enormous contribution to the area of Infectious Diseases in the country. Together, we are committed to continuing to work towards the growth and consolidation of this society. All of you affiliated members are responsible not only for the creation of this society but for the success that has been achieved throughout these 25 years of its existence. It is impossible to name everyone here who has helped and supported this Society, but we must thank Professor Ricardo Veronesi (in memoriam), and by doing so we extend our gratitude to all of you who have contributed directly or indirectly to the consolidation of The Brazilian Society of Infectious Diseases, formerly the Sociedade Brasileira de Infectologia.

\author{
João Silva de Mendonça \\ President - BSID \\ Roberto Badaró and Anastácio Q. Sousa \\ Editors-in-Chief-BJID
}

